\title{
Impact of Laser Frequency Noise in Coherent Population Trapping with Cold Atoms
}

\author{
Gregory W. Hoth, Rachel Elvin, Michael W. Wright, \\ Ben Lewis, Aidan S. Arnold, Paul F. Griffin, \\ and Erling Riis \\ Department of Physics, SUPA, University of Strathclyde \\ Glasgow, United Kingdom \\ gregory.hoth@strath.ac.uk
}

\author{
Florian Gruet, Christoph Affolderbach, \\ and Gaetano Mileti \\ Laboratoire Temps-Fréquence, Institut de Physique \\ Université de Neuchâtel \\ Neuchâtel, Switzerland
}

\begin{abstract}
Laser-cooled atoms and coherent population trapping (CPT) are promising tools for realizing a compact microwave frequency reference with excellent stability. To realize a high performance device, it is necessary to understand and minimize all sources of technical noise. Here, we investigate the role of laser frequency noise in cold-atom CPT with an apparatus based on the grating magneto-optical trap (GMOT). We compare the performance of our setup with an external cavity diode laser (ECDL) and a distributed feedback diode laser (DFB). With the DFB, laser frequency noise is one of the dominant noise sources in our system. With the ECDL, it is significantly reduced. We also report frequency stability measurements of our apparatus with a short-term Allan deviation $\sigma_{\mathrm{y}}(\tau) \approx 3 \times 10^{-11} / \sqrt{ } \tau$ up to $\tau=10 \mathrm{~s}$.
\end{abstract}

Keywords-Coherent population trapping, laser cooling, coldatom clock, microwave frequency standard.

\section{INTRODUCTION}

Over the last three decades, microwave frequency references based on hyperfine transitions in laser-cooled atoms have achieved stability below $1 \times 10^{-13} / \sqrt{ } \tau$ with the ability to average down to the level of a few times $10^{-16}[1]$. The majority of coldatom microwave frequency references utilize a microwave cavity to probe the atomic resonance. This approach works very well, but the use of a cavity also imposes constraints on the size, optical access, and environmental sensitivities of these systems. These constraints can be exchanged for a different set of challenges by use of coherent population trapping (CPT). In $\mathrm{CPT}$, the microwave atomic coherence is optically pumped by use of a bichromatic laser [2],[3]. This approach has enabled compact physics packages in clocks based on thermal atoms [4], and it is a promising route to explore for compact devices based on laser-cooled atoms [5]-[9].

It is straightforward to come up with examples where an ideal cold atom CPT clock could achieve a stability on the order of $10^{-13} /{ }_{\tau}$. For example, a system with quantum projection noise limited detection of $N=10^{7}$ atoms, an interrogation time of $T=15 \mathrm{~ms}$, and a cycle time of $100 \mathrm{~ms}$ would have an expected short-term stability of $\sigma_{\mathrm{y}}(\tau) \approx 2 \times 10^{-13} / \sqrt{ } \tau[10]$. In

This work was financially supported by EPSRC (EP/M013294/1), the UK Quantum Technology Hub for Sensors and Metrology, the National Physical Laboratory, and ESA (project no. 4000120052/17/NL/BJ and 4000108465/ RFQ/3-13789/13/NL/PA), and the Swiss Space Center (EPFL-ESA/LS$\mathrm{COO} 17)$ practice, it is challenging to reach these hypothetical stabilities. For comparison with experiment, a cold atom CPT system has recently achieved a short-term stability of $\sigma_{\mathrm{y}}(\tau) \approx 1.3 \times 10^{-11} /{ } \tau$ [9]. In pursuit of the long-term goal of realizing a cold atom CPT system with a stability on the order of $10^{-13} / \sqrt{ } \tau$, it is interesting to study the limits to the signal-to-noise ratio (SNR) in these systems. One important limit to the SNR in many atomic sensors is laser frequency noise [7]-[14]. Here, we study the role of laser frequency noise in our cold-atom CPT apparatus and show that we can achieve a short term stability of $\sigma_{\mathrm{y}}(\tau) \approx 3 \times 10^{-11} / \sqrt{ } \tau$ up to $\tau=10 \mathrm{~s}$.

\section{APPARATUS}

In order to investigate the achievable performance with cold atoms and CPT, we employ an apparatus that combines a compact magneto-optical trap formed by an optical diffraction grating (GMOT) [15] with lin $\perp$ lin CPT [16]-[19]. Our system has been described in [20]-[22], and it is shown schematically in Fig. 1. We realize a CPT resonance on the $\mathrm{D}_{1}$ line of ${ }^{87} \mathrm{Rb}$ using the $F^{\prime}=2$ excited state. The light used to excite the resonance is obtained by splitting the light from one laser into two paths. In one path, the light is modulated by an electro-optic modulator $(\mathrm{EOM})$ driven at a frequency $\mathrm{f}_{\mathrm{EOM}} \approx 7.0 \mathrm{GHz}$. In the other path, the light is frequency shifted by an acousto-optic modulator (AOM 1) driven at a frequency $\mathrm{f}_{\mathrm{AOM}} \approx 0.2 \mathrm{GHz}$. The beams are recombined on a polarizing beam splitter (PBS), and directed through a second AOM (AOM 2). After this second $\mathrm{AOM}$, the frequency shifted beam from AOM 1 and the firstorder EOM sideband form a pair of resonant, orthogonallypolarized laser modes with a frequency difference close to the hyperfine splitting of ${ }^{87} \mathrm{Rb}$. The combined beam also contains other off-resonant laser sidebands from the EOM. The laser is stabilized using a saturated absorption spectrometer (SAS) which incorporates a third AOM to make the lock point tunable [20] (not shown in Fig. 1).

With this approach, we are able to implement the $\operatorname{lin} \perp$ lin CPT scheme with a single laser source. This is an advantage for our long-term goal of developing a compact device, but this approach also introduces a significant source of phase noise due to the separated paths of the two CPT components [23]. In order to suppress this phase noise, we implement a phase-locked loop (PLL) by detecting the phase of the beatnote between the light 


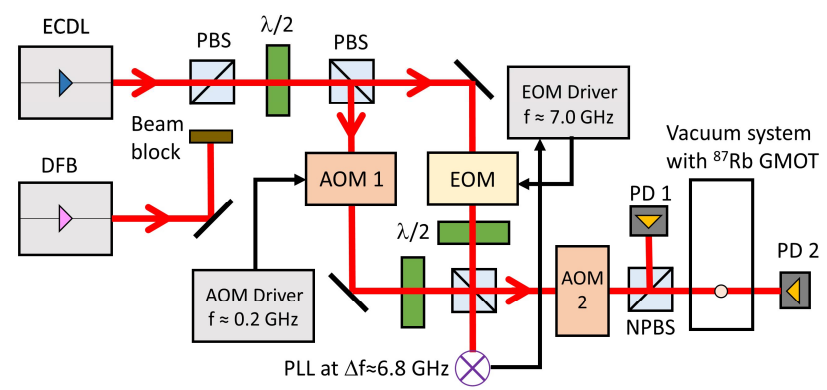

Fig. 1. A schematic diagram of our apparatus. By moving the beam block, we are able to switch between the two laser sources. Only one of the lasers is used at a time. The two lasers were coupled into a common single-mode fiber before the first PBS shown in the diagram in order to ensure the beam paths were the same for both lasers. By engaging or removing the phaselocked loop (PLL) feeding back to the EOM driver, we are able to measure the effects of the phase noise due to the separated optical paths. The frequency difference between the driver for AOM 1 and the EOM is $\Delta \mathrm{f}$, and PD denotes photodiodes.

from AOM 1 and the first-order sideband from the EOM and feeding back to our EOM driver. The implementation of the PLL is described in more detail in [22].

We obtain a clock signal with a Ramsey CPT sequence [5][9],[16]-[18] that consists of a MOT loading stage, followed by a first pulse to optically pump the atoms into a dark state, then a free evolution time with duration $T$ and, finally, a second readout pulse. For the experiments described here, the MOT was loaded for about $70 \mathrm{~ms}$, and an estimated steady state atom number of $N \geq 9 \times 10^{6}$ was achieved by recapturing the cold atoms from cycle to cycle [6],[22],[24]. The pumping and readout pulses were both $0.3 \mathrm{~ms}$ long. The EOM was driven so that there were approximately equal powers in the carrier and the first order sidebands, and the powers of the two lin $\perp$ lin CPT components were approximately equal. The peak intensity of each single optical frequency component driving the CPT was approximately $0.2 \mathrm{~mW} / \mathrm{cm}^{2}$.

In the Ramsey CPT sequence, the relative phase between the local oscillator (LO) and the atomic coherence manifests as an optical pumping transient at the beginning of the read-out pulse [3]. If the LO is in phase with the atomic coherence, the atoms are still in the dark state, and so there is no optical pumping. However, if the LO is out of phase with the atomic coherence, the atoms will be in a bright state and they will be optically pumped back to the dark state. We detect these optical pumping transients using a double-ratio technique to suppress laser intensity noise [6]-[8]. For the first ratio, we split the combined beam on a non-polarizing 50/50 beam splitter (NPBS). One output from the splitter is detected on a photodiode (PD 1), while the other beam propagates through the cold atoms before being detected on a second photodiode (PD 2). By taking the ratio of these two signals, we measure the transmission of the atoms. For the second ratio, we take advantage of the transient nature of the Ramsey CPT signals. In particular, we integrate the transmission for $28 \mu \mathrm{s}$ at the beginning of the read-out pulse and divide it by the integrated transmission after the optical pumping transient has settled. This second ratio helps to cancel out noise that is not correlated between the two photodiodes.

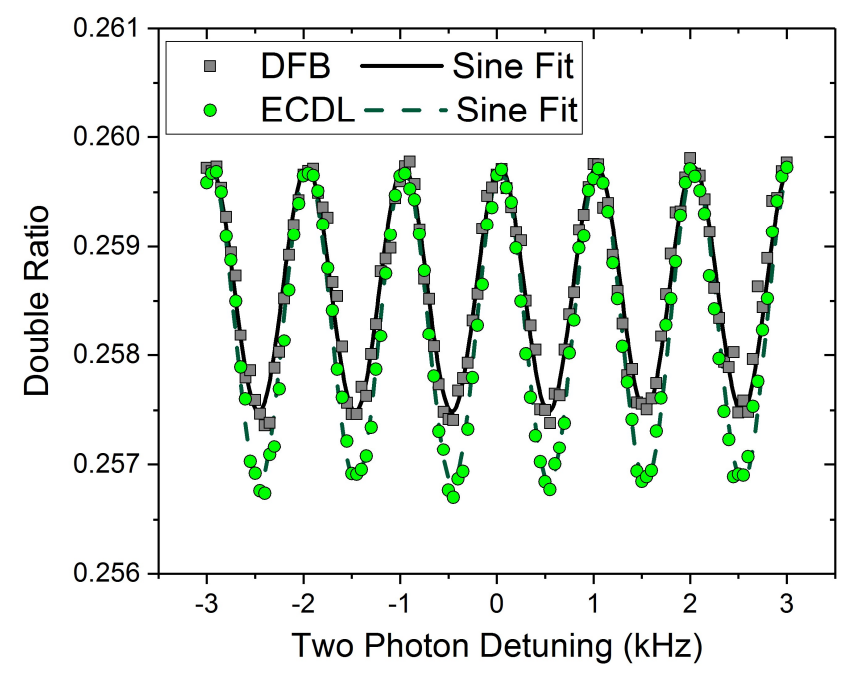

Fig. 2. Examples of $\mathrm{T}=1 \mathrm{~ms}$ Ramsey fringes measured in our apparatus with both the DFB and the ECDL lasers. For the DFB, the fit amplitude (peak-peak) is $\mathrm{a}=2.24 \pm 0.04 \times 10^{-3}$. For the ECDL, $\mathrm{a}=2.88 \pm 0.04 \times 10^{-3}$. The central fringe offset is about $40 \mathrm{~Hz}$, dominated by the quadratic Zeeman shift for the relatively large bias field of $B \approx 260 \mathrm{mG}$ used for these measurements. The average value of the double-ratio signal is mostly set by the durations of the integration regions. For these data, the signal region at the beginning of the detection pulse was $28 \mu \mathrm{s}$ long, and the normalization region near the end of the detection pulse was $100 \mu$ s long. Each point corresponds to the transmission of a single sample of cold atoms from one run of the experiment.

By scanning the EOM frequency, we are able to quantify the characteristics of our CPT resonance and observe Ramsey fringes. With a single CPT pulse and both CPT components on optical resonance, the peak absorption is about $2 \%$. The contrast of the $\mathrm{m}_{\mathrm{F}}=0 \mathrm{CPT}$ resonance was greater than $50 \%$ (with respect to the maximum one-photon optical absorption). After optimizing the upgraded system described below, we have obtained CPT contrasts of about $75 \%$. Examples of Ramsey fringes obtained in our apparatus are shown in Fig. 2.

For this study, we compared the performance of our apparatus with two lasers: a frequency-stabilized laser head [25] developed at the Universite de Neuchâtel based on a distributed feedback diode laser (DFB) and a commercial external cavity diode laser (ECDL). The DFB had a measured free-running linewidth of $2.4 \pm 0.9 \mathrm{MHz}$ (full width at half maximum, FWHM). The ECDL had a specified RMS frequency noise of less than $150 \mathrm{kHz}$ for the stabilized laser, which corresponds to a FWHM of $350 \mathrm{kHz}$ for a Gaussian lineshape [26]. A beatnote between the two free-running lasers had a linewidth of $3.1 \pm 0.7 \mathrm{MHz}$ and an analysis of the stabilized ECDL frequency noise spectrum [27] estimated an ECDL FWHM of about $250 \mathrm{kHz}$ in reasonable agreement with the specifications (neglecting the modulation described below). The time scale for the measured linewidths was $1 \mathrm{~ms}$ or longer. In the CPT experiments, both lasers were current modulated to obtain the spectroscopy signals used to stabilize the laser frequency. The DFB was modulated at a frequency of $50 \mathrm{kHz}$ while the ECDL was modulated at a frequency of $250 \mathrm{kHz}$. The modulation depth was not measured for this initial noise study, 
but measurements after the study and on similar laser systems indicate that the modulation depth was greater than $1 \mathrm{MHz}$ (peak to peak) for both lasers. In future work, we plan to investigate the contributions of intrinsic laser noise and the characteristics of the frequency modulation in more detail.

With the set up shown in Fig. 1, it was straightforward to measure CPT signals with both lasers. We found that the DFB laser produced a broadening of the optical absorption line and a decrease in the peak absorption, which is expected when the laser linewidth becomes comparable to the linewidth of the atomic transition [11]. As a result, the DFB laser produced Ramsey fringes with a lower amplitude then the ECDL. For the data shown in Fig. 2, the fringes were measured approximately 1 hour apart, and the fringes produced with the DFB laser were $30 \%$ smaller than the fringes with the ECDL.

\section{LASER FREQUENCY NOISE IN COLD-ATOM CPT}

In order to explore the influence of laser frequency noise in our apparatus, we measured the noise on our Ramsey fringe signal as a function of the optical detuning of the CPT-driving light. In this configuration, the two CPT-driving frequency components are detuned from optical resonance by a common offset while the frequency difference between the two components is still nearly equal to the hyperfine splitting. The optical detuning was varied by changing the drive frequency of the AOM in the SAS used to lock the CPT laser. We also measured the noise at different phases of the Ramsey fringe in order to probe the contribution of different noise sources [7]. By measuring the noise at the top of the Ramsey fringes where the atomic coherence is in phase with the CPT light, we were able to quantify the effect of laser frequency noise on the scattering that occurs due to finite CPT contrast. By measuring the noise at the bottom of the Ramsey fringes, we were able to probe the effect of laser frequency noise on the optical pumping transients. Finally, by measuring the noise on the side of the Ramsey fringes, we were able to see the effect of the phase noise due to the separated paths in Fig. 1.

We determined the noise floor for these measurements by running the experiment without any cold atoms [7]. This technical floor is due to a combination of the noise of the detection electronics, photon shot noise, and laser intensity noise that is not cancelled out by the double ratio [7]. Measurements of the noise floor as a function of the optical detuning show that it is well approximated by a flat line over the range of interest here. Repeated measurements suggest the uncertainty in the floor is about $5 \%$. The technical noise floor measured with the DFB and the ECDL differed by $8 \%$. For the noise measurements with cold atoms, we chose to work with $T=1 \mathrm{~ms}$ Ramsey fringes to maximize the signal amplitude and minimize the importance of the technical noise floor.

We quantified the noise by running the experiment at four two-photon detunings, controlled by the EOM frequency, which corresponded to the top, bottom, and left and right sides of the Ramsey fringes. For each fringe position, we repeated the experimental sequence approximately 120 times and calculated the standard deviation of the measured signals. Then, we repeated this process several times for each optical detuning.

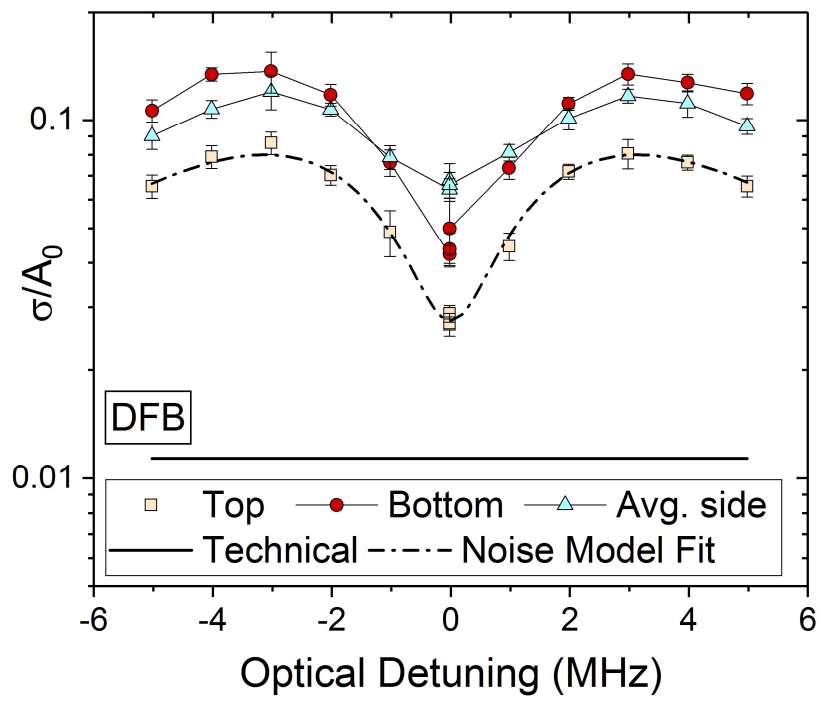

Fig. 3. Measured noise $(\sigma)$ with the DFB as a function of the optical detuning and the Ramsey fringe position with $T=1 \mathrm{~ms}$, normalized to the fringe amplitude near resonance $\left(\mathrm{A}_{0}\right)$. The noise is estimated as the standard deviation of 120 runs, and the error bars are estimated by repeating this 7 times for each point. The solid line indicates the noise floor measured without cold atoms, while the dashed line represents a fit of (2) to the noise measured at the top of the fringe. The best fit parameters are $\alpha / \mathrm{A}_{0}=24.5 \pm 1.3 \mathrm{MHz}^{2}, \Gamma=10.8 \pm 0.3 \mathrm{MHz}$ and $\sigma_{\delta}=0.79 \pm 0.03 \mathrm{MHz}$. The uncertainties were estimated by the nonlinear least squares fitting routine.

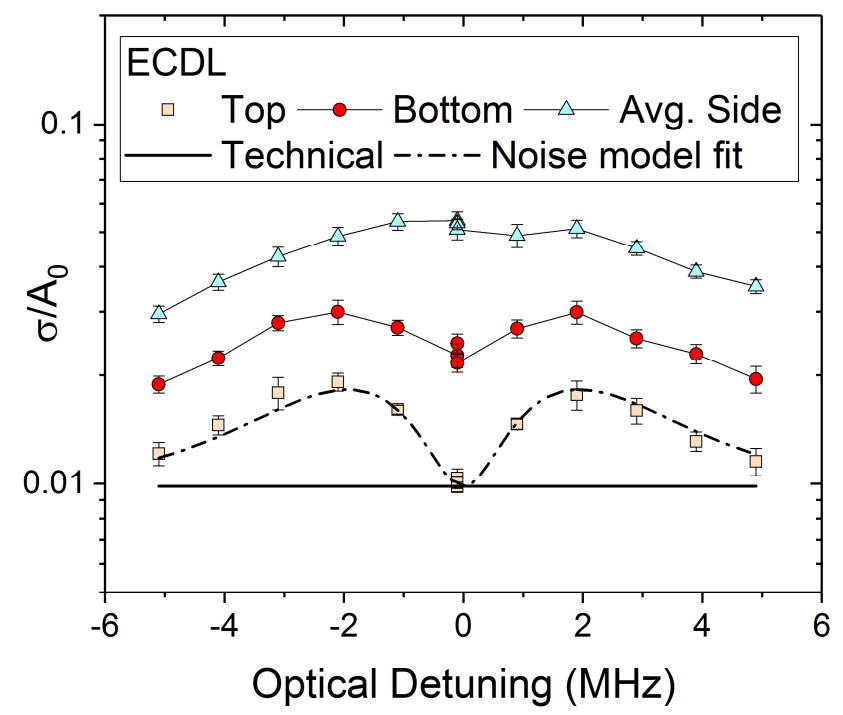

Fig. 4. Measured noise $(\sigma)$ with the ECDL as a function of the optical detuning and the Ramsey fringe position with $T=1 \mathrm{~ms}$, normalized to the fringe amplitude near resonance $\left(\mathrm{A}_{0}\right)$. The noise is estimated as the standard deviation of 120 runs, and the error bars are estimated by repeating this 7 times for each point. The solid line indicates the noise floor measured without cold atoms, while the dashed line represents a fit of (2) to the noise measured at the top of the fringe. The best fit parameters are $\alpha \sigma_{\delta} / \mathrm{A}_{0}=0.9 \pm 0.1 \mathrm{MHz}^{3}, \Gamma=6.7 \pm 0.2 \mathrm{MHz}$. The uncertainties were estimated by the nonlinear least squares fitting routine

The results of our initial measurement campaign with the two lasers are summarized in Figs. 3 (DFB) and 4 (ECDL). For this initial study, the PLL in Fig. 1 was not implemented. To plot the measured noise $(\sigma)$ in sensible units, we normalize it by the Ramsey fringe amplitude (peak-peak) on resonance $\left(\mathrm{A}_{0}\right)$. For a 
full accounting of the SNR, it is important to remember that the Ramsey fringe amplitude also decreases as the optical detuning is moved away from resonance.

Looking at Figs. 3 and 4, one can see several interesting points of comparison between the two datasets. First, consider the noise measurements on the top of the Ramsey fringes. With both lasers, we see an m-like shape [28] that is characteristic of laser frequency noise converted to amplitude noise [11],[28]. Although this conversion is a non-linear process [11], it can often be modeled by treating the laser frequency noise as a small fluctuation in the optical detuning and Taylor expanding the atomic response [12]-[14]. We apply these ideas in this case by fitting our top of the fringe measurements to a function built from derivatives of a Lorentzian absorption line. The Lorentzian is parameterized as

$$
L(\delta)=\frac{\alpha}{\delta^{2}+(\Gamma / 2)^{2}},
$$

where $\delta$ is the optical detuning, $\alpha$ is an overall amplitude and $\Gamma$ is the FWHM. Following a suggestion by E. Rocco et al. [14], we fit the noise measurements to a function of the form

$$
\sigma_{\text {top }}^{2}=\sigma_{0}^{2}+\left(\frac{\partial L}{\partial \delta}\right)^{2} \sigma_{\delta}^{2}+\left(\frac{\partial^{2} L}{\partial \delta^{2}}\right)^{2} \sigma_{\delta}^{4} / 2
$$

where $\sigma_{0}$ represents a noise floor that is unrelated to laser frequency noise, and $\sigma_{\delta}$ represents the fluctuations in the laser frequency. In principle, it should be possible to calculate $\sigma_{\delta}$ by combining a measurement of the laser frequency noise power spectrum with knowledge of how the converted frequency noise is filtered by the photodiode bandwidth and the detection windows [12]. Here, we treat $\alpha, \Gamma$, and $\sigma_{\delta}$ as free parameters while the noise floor $\sigma 0$ is constrained to the measured technical noise floor.

As shown in Figs. 3 and 4, this model provides a good description of the data. However, it is necessary to use some care in the fitting procedure due to couplings between the parameters. In particular, only the second derivative term makes a distinction between $\alpha$ and $\sigma$. The contribution of the second derivative term also falls away rapidly as a function of $\delta$ so that it is mainly significant near $\delta \approx 0$, which means it is hard to distinguish from the noise floor, $\sigma_{0}$. In the case of the DFB, we concluded that the large increase in noise above the no-atoms noise floor is mainly due to the increased frequency noise of the laser, and this makes it possible to fit the second derivative term directly. For this fit, we fixed $\sigma 0$ at the measured technical noise floor and fit the remaining two terms in (2) to produce the curve shown in Fig. 3. For the ECDL, the data near resonance is close to the technical noise floor, which means the third term in (2) cannot be fit reliably. In this case, we can fit the first derivative term in (2) by treating the product $\alpha \sigma_{\delta}$ as a single parameter. The noise floor $\sigma 0$ can either be fit or fixed at the measured noise floor without much effect on the results.

Given the success of the fit function in (2) for the top of the fringe data, it is natural to try to identify the frequency discriminator responsible for frequency noise conversion in this case. One natural candidate to investigate is the residual absorption on the CPT resonance. Preliminary measurements of the absorption on the CPT resonance as a function of the optical detuning are consistent with the noise model fits, but more work is needed to confirm this connection.

We now turn to consider the data on the bottom and side of the Ramsey fringes. Here, our analysis is more qualitative in character. On the bottom of the Ramsey fringes, the measured noise also shows an m-like shape, which is similar to the top of the fringe noise data for both the DFB and the ECDL. There is clearly more noise on the bottom of the fringe compared to the top, which suggests that there is another path for laser frequency noise conversion. We plan to investigate this in more detail in future work. With the ECDL, the measurements near resonance suggest that there is another source adding noise to the bottom of the fringe. After some investigation, we found that the increase in noise at the bottom of the fringe near zero optical detuning was due to atom number fluctuations driven by an intensity instability in the MOT light.

On the side of the fringe, we see a clear difference between the two lasers. With the DFB, we see something like the mshape that is present on the top and bottom of the fringe, which suggests laser frequency noise also plays a role in this case. With the ECDL data, there is no sign of laser frequency noise conversion on the side of the fringe. Our interpretation is that the noise is dominated by the phase noise due to the separated paths in this case. If we divide the measured noise by the Ramsey fringe amplitude as a function of the optical detuning, we find that the SNR on the side of the fringe is nearly independent of the optical detuning as expected for phase noise.

There is one detail to note about the DFB data which is not shown in Fig. 3. With the DFB laser, there was a significant asymmetry in the noise measurements made on the left and right sides of the Ramsey fringe. A smaller asymmetry may also be present in the ECDL data, but it is not distinct from the estimated measurement uncertainty. As shown in Fig. 3, the asymmetry nearly vanishes when the noise measurements from the left and the right side are averaged together. Near the peak in the measured noise curve, the difference between the left and the right side was about $25 \%$ of the average noise. The source of this asymmetry is unknown. One possible explanation is that some of the phase noise is actually due to laser frequency noise, which is converted to phase noise by the mismatched optical paths in Fig. 1 [23]. In this case, there could be a coherence between the phase noise and other sources of frequency noise conversion, which would lead to either constructive or destructive interference depending on the slope of the Ramsey fringe.

After this initial noise assessment, we made several improvements to our apparatus to reduce the noise. In particular, we implemented the PLL described in [22] to reduce the phase noise, improved the stability of the MOT light to reduce the atom number noise, and improved the detection system to reduce the technical noise floor. After these upgrades, we repeated the measurement of noise versus fringe position and optical detuning with the ECDL and obtained the results shown in Fig. 5. We see significant reductions in the noise 


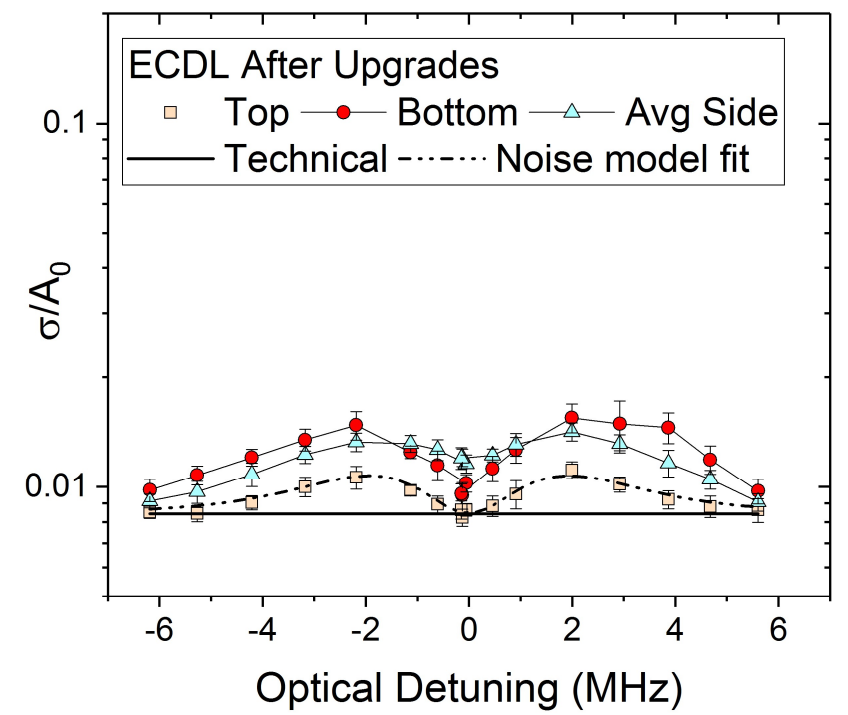

Fig. 5. Measured noise $(\sigma)$ with the ECDL as a function of the optical detuning and the Ramsey fringe position with $T=1 \mathrm{~ms}$, normalized to the fringe amplitude near resonance $\left(\mathrm{A}_{0}\right)$. The noise is estimated as the standard deviation of 120 runs, and the error bars are estimated by repeating this 12 times for each point. The solid line indicates the noise floor measured without cold atoms, while the dashed line represents a fit of (2) to the noise measured at the top of the fringe. The best fit parameters are $\alpha \sigma_{\delta} / \mathrm{A}_{0}=0.37 \pm 0.04 \mathrm{MHz}^{3}, \Gamma=6.7 \pm 0.3 \mathrm{MHz}$. The uncertainties were estimated by the nonlinear least squares fitting routine

compared to the original measurement in Fig. 3. The reductions in noise at the bottom and side of the fringe were expected from the upgrades. The reduction in noise at the top of the fringe is consistent with the measured reduction in the scattering on the CPT resonance. This reduction is due to a decrease in the total absorption (due to a smaller number of atoms, which is due to less power in the MOT beam) and an increase in the CPT contrast due to an improved optimization of our system.

\section{Stability MEASUREMENTS}

In order to probe the stability of the apparatus, we have also measured the frequency stability of the central Ramsey fringe with $T=10 \mathrm{~ms}$. The results are summarized in Fig. 6 . In the two laser measurement campaign, we found that with the ECDL (DFB), the short-term Allan deviation was $\sigma_{\mathrm{y}}(\tau) \approx 6.3 \times 10^{-11} / \sqrt{ } \tau$ $\left(10 \times 10^{-11} / \sqrt{ } \tau\right)$ up to $\tau \approx 10 \mathrm{~s}$. After implementing the upgrades described in the previous section, we obtained a short term Allan deviation of $\sigma_{\mathrm{y}}(\tau) \approx 3.0 \times 10^{-11} / \sqrt{ } \tau$. With the upgraded system, we are also able to integrate down a little bit more. This is due to the addition of the PLL in Fig. 1 and improvements in managing the influence of variations in the magnetic environment on the cold atoms.

Our current system is unshielded, which means magnetic gradients and $50 \mathrm{~Hz}$ noise are significant problems. These imperfections can be suppressed by operating with a relatively large magnetic bias field of about $260 \mathrm{mG}$. For the measurement with the upgraded system in Fig. 6, we reduced the dc magnetic bias field to about $100 \mathrm{mG}$. By synchronizing the experiment with the mains power frequency to suppress the influence of $50 \mathrm{~Hz}$ noise, we were able to achieve $\mathrm{SNR} \approx 45$ in a single shot at $T=10 \mathrm{~ms}$ with the lower magnetic bias field. In

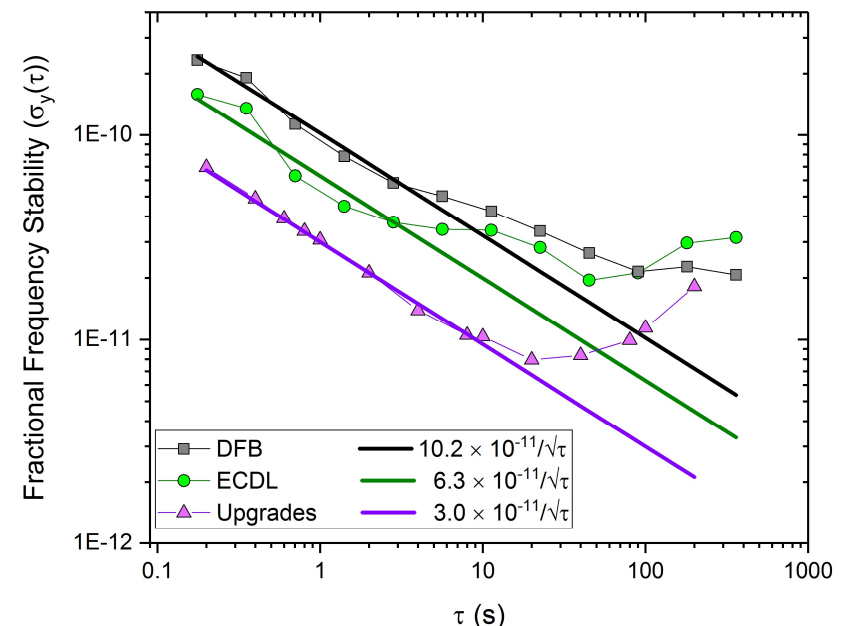

Fig. 6. Measured Allan deviations for our apparatus with $T=10 \mathrm{~ms}$ Ramsey fringes and several different configurations. The Allan deviation is estimated by alternating between the left and right side of the central Ramsey fringe for about half an hour. From these data, we estimate the difference between the frequency synthesized by our RF system and the atomic resonance frequency. The RF system is referenced to a GPS disciplined crystal oscillator that is specified to have a fractional frequency stability $\sigma_{\mathrm{y}} \leq 1 \times 10^{-12}$ for all $\tau$.

this configuration, the measured noise was within $20 \%$ of the technical noise floor at all three fringe positions.

We are currently investigating the sources of instability around $\sigma_{\mathrm{y}} \approx 1 \times 10^{-11}$. The two most likely candidates are magnetic field variations and Doppler shifts. These can be suppressed by adding a magnetic shield to the experiment and by retro-reflecting the CPT beam [6].

\section{CONCLUSIONS}

We have identified several contributions to the SNR in our apparatus by varying the optical detuning and the Ramsey fringe position. Building on the results obtained through this noise assessment, we have achieved an $\mathrm{SNR} \approx 80$ on the side of the $T=1 \mathrm{~ms}$ fringes. The limits to the SNR in the upgraded system are currently under investigation. The different shapes of the noise curves between the side of the fringe and the top and bottom of the fringe shown in Fig. 5 suggest it is unlikely that the conversion of laser frequency noise to amplitude noise is the dominant limit to the SNR in this case.

Alongside this initial noise characterization, we have also quantified stability of our apparatus and measured a best case stability of $\sigma_{\mathrm{y}}(\tau) \approx 3.0 \times 10^{-11} / \sqrt{ } \tau$ to an averaging time of $10 \mathrm{~s}$. In the short term, we hope to improve the stability of our apparatus at averaging times longer than $10 \mathrm{~s}$ by implementing a magnetic shield and retro-reflection [6]. In the long term, we plan to continue to improve the SNR towards the quantum projection noise limit and develop a compact, cold atom CPT clock.

\section{ACKNOWLEDGMENT}

The authors gratefully acknowledge Leo Hollberg for useful technical discussions. The data supporting this publication can be accessed at https://doi.org/10.15129/caa1782a-4e72-438ba5bc-97425e68154b.

\section{REFERENCES}


[1] S. Weyers et al., "Advances in the accuracy, stability, and reliability of the PTB primary fountain clocks," Metrologia, vol. 55, pp. 789-805, Oct. 2018.

[2] J. Vanier, "Atomic clocks based on coherent population trapping: a review," Appl. Phys. B, vol. 81, pp. 421-442, July 2005.

[3] V. Shah and J. Kitching, "Advances in coherent population trapping for atomic clocks," in Advances in Atomic, Molecular, and Optical Physics, vol. 59, E. Arimondo, P. Berman, and C. C. Lin, Eds. Academic Press, 2010, pp. 21-74.

[4] S. Knappe et al., "A microfabricated atomic clock,” Appl. Phys. Lett., vol. 85, pp. 1460-1462, Aug. 2004.

[5] X. Chen, G.-Q. Yang, J. Wang, and M.-S. Zhan, "Coherent population trapping-ramsey interference in cold atoms," Chin. Phys. Lett., vol. 27, 113201, Aug. 2010.

[6] F.-X. Esnault, et al., "Cold-atom double- $\Lambda$ coherent population trapping clock” Phys. Rev. A, vol. 88, 042120 Oct. 2013.

[7] E. Blanshan, "Development of a compact cold-atom atomic clock based on coherent population trapping," Ph.D. dissertation, Dept. Phys., Univ. Colorado, Boulder, USA, 2014.

[8] X. Liu, E. Ivanov, V. I. Yudin, J. Kitching, and E. A. Donley, "Low-drift coherent population trapping clock based on laser-cooled atoms and high coherence excitation fields" Phys. Rev. Appl., vol. 8, 054001, Nov. 2017.

[9] X. Liu, V. I. Yudin, A. V. Taichenachev, J. Kitching, E. A. Donley, "High contrast dark resonances in a cold-atom clock probed with counterpropagating circularly polarized beams," Appl. Phys. Lett., vol. 111, 224102, Nov. 2017.

[10] G. Santarelli et al., "Quantum projection noise in an atomic fountain: a high stability cesium frequency standard," Phys. Rev. Lett., vol. 82, pp. 4619-4622, June 1999.

[11] J. C. Camparo and J. G. Coffer, "Conversion of laser phase noise to amplitude noise in a resonant atomic vapor: the role of laser linewidth," Phys. Rev. A, vol. 59, pp. 728-735, Jan. 1999.

[12] F.-X. Esnault, "Etude des performances ultimes d'une horloge compacte à atomes froids : optimisation de la stabilité court terme" Ph.D. dissertation, Université Pierre et Marie Curie, Paris, France, 2009.

[13] J. Kitching, H. G. Robinson, L. Hollberg, S. Knappe, and R. Wynands, "Optical-pumping noise in laser-pumped, all-optical microwave frequency references,” J. Opt. Soc. Am. B, vol. 18, pp. 1676-1683, Nov. 2001.

[14] E. Rocco et al., "Fluorescence detection at the atom shot noise limit for atom interferometry," New J. Phys., vol. 16, 093046, Sept. 2014.

[15] C. C. Nshii et al., "A surface-patterned chip as a strong source of ultracold atoms for quantum technologies," Nature Nanotech., vol. 8, pp. 321-324, May 2013.

[16] T. Zanon et al., "High contrast ramsey fringes with coherent-populationtrapping pulses in a double lambda atomic system," Phys. Rev. Lett., vol. 94, 193002, May 2005.

[17] R. Boudot, S. Guérandel, E. de Clercq, N. Dimarcq, and A. Clairon, "Current status of a pulsed CPT Cs cell clock," IEEE T. Instr. Meas., vol. 58, pp. 1217-1222, Apr. 2009.

[18] O. Kozlova, J.-M. Danet, S. Guérandel, and E. de Clercq, "Limitations of long-term stability in a coherent population trapping Cs clock," IEEE T. Instr. Meas., vol. 63, pp. 1863-1870, July 2014.

[19] Z. Warren, M. S. Shariar, R. Tripathi, and G. S. Pati, "Experimental and theoretical comparison of different optical excitation schemes for a compact coherent population trapping Rb vapor clock," Metrologia, vol. 54 pp. 418-431, June 2017.

[20] J. P. McGilligan, "Micro-fabricated diffractive optics for quantum sensors and atomic clocks," Ph.D. dissertation, Dept. Phys., Univ. Strathclyde, Glasgow, UK, 2017.

[21] R. Elvin et al., "Raman-ramsey CPT with a grating magneto-optical trap," in Proc. 2018 European Frequency and Time Forum, 2018, pp. 61-64, doi:10.1109/EFTF.2018.8408998.

[22] G. W. Hoth et al., "Towards a compact atomic clock based on coherent population trapping and the grating magneto-optical trap," in Proc. SPIE 10934, Optical, Opto-Atomic, and Entanglement-Enhanced Precision Metrology, vol. 10934 2019, doi: 10.1117/12.2516612.
[23] W. Li, X. Pan, N. Song, X. Xu, and X. Lu, "A phase-locked laser system based on double direct modulation technique for atom interferometry", Appl. Phys. B., vol. 123, 54 Feb. 2017.

[24] A. V. Rakholia, H. J. McGuinness, and G. W. Biedermann, "Dual-axis, high-data-rate atom interferometer via cold ensemble exchange," Phys. Rev. Appl., vol. 2, 054012 Nov. 2014.

[25] F. Gruet et al., "Compact and frequency stabilized laser heads for rubidium atomic clocks," in Proc. SPIE 10564, Int. Conf. Space Optics, 2012, doi: 10.1117/12.2309135.

[26] L. D. Turner, K. P., Weber, C. J. Hawthorn, and R .E. Scholten et al., "Frequency noise characterisation of narrow linewidth diode lasers", Opt. Commun., vol. 201, pp. 391-397 Jan. 2002.

[27] G. D. Domenico, S. Schilt, and P. Thomann, "Simple approach to the relation between laser frequency noise and laser line shape," Appl. Optics, vol. 49, pp. 4801-4807, Sept. 2010.

[28] M. Bahoura and A. Clairon, "Diode-laser noise conversion in an optically dense atomic sample, ” Opt. Lett., vol. 26, 926-928, Jan. 2001. 